\title{
Strain estimation in the carotid artery from ultrasonic wall tracking: a multiphysics model study
}

\author{
Abigail Swillens \\ Gianluca De Santis \\ Patrick Segers \\ IBiTech-bioMMeda
}

Ghent University, Ghent, Belgium

Email: abigail.swillens@ugent.be

\author{
Lasse Lovstakken \\ Department of Circulation and Medical Imaging \\ NTNU, Trondheim, Norway \\ St. Olavs University Hospital, Trondheim, Norway
}

\author{
Joris Degroote \\ Jan Vierendeels \\ Department of flow, heat \\ and combustion mechanics \\ Ghent University, Ghent, Belgium
}

\begin{abstract}
Non-invasive assessment of large arteries is important for cardiovascular screening, with the carotid artery being a preferred measurement location. Ultrasonic (US) wall tracking allows measuring arterial motion and distensibility (kinematics), but it is unclear to what extent the technique can be used to derive arterial strain (mechanics). For this purpose, we used a multiphysics model to assess the accuracy of carotid strain estimates derived from a 1D ultrasonic wall tracking algorithm. Distension estimates were then obtained by using tracking points at various depths within the wall, which were further processed to assess radial and circumferential strain. The simulated data demonstrated that circumferential strain can be estimated with reasonable accuracy (especially for the common carotid artery and at the lumen-intima and media-adventitia interface), but the technique does not allow to reliably assess intra-arterial radial strain. These findings were supported by in-vivo data of 10 healthy adults, showing similar circumferential and radial strain profiles throughout the arterial wall. We conclude that these deviations are present due to the complex 3D vessel wall deformation and the presence of specular reflections, with the error depending on the phase in the cardiac cycle and the scanning location.
\end{abstract}

\section{INTRODUCTION}

In the search for improved detection methods for cardiovascular disease, arterial stiffness has proven to be an early marker of atherosclerosis. Several non-invasive methods using ultrasound have been developed to measure arterial stiffness. A common method is by measurement of pulse wave velocity (PWV), i.e. the velocity with which the arterial pressure and flow waves travel through the cardiovascular system. When using ultrasound, one of the ways to capture the propagation of the arterial pulse along a short arterial segment is by measuring the propagation of the diameter distension wave along several parallel scanlines at a temporal resolution which is high enough to track the fastly propagating wave front (wave speed is in the order of 3-10 m/s) [1]. Further, ultrasonic strain imaging using elastographic methods has been extensively investigated to measure axial and lateral arterial strain, a challenging task due to the limited resolution compared to the vessel wall dimensions [2]. Another revolutionary technique showing much promise is supersonic shear wave imaging, which generates shear waves in the arterial wall through the acoustic radiation force and subsequently estimates the shear wave speed, inherently related to the mechanical properties of the artery [3]. Finally, mechanical properties can also be measured through arterial wall distension, which is the focus of this paper. Ultrasonic techniques have been successfully developed to assess vessel diameter distension during the cardiac cycle by estimating the tissue velocity [4] (wall tracking methods).

However, the above-mentioned applications have not found their way yet to clinical practice. As such, we will assess in this work the accuracy of ultrasonic estimation of radial and circumferential strain and related material properties as derived from a wall tracking algorithm, using both multiphysics simulations and in-vivo data. The performance of wall tracking algorithms was previously analyzed in a simplified tube configuration in order to ease the interpretation of the measured distensions $(\Delta D)$ and the corresponding circumferential strain $(\Delta D / D)$ estimates [5]. We demonstrated that distension and circumferential strain estimates as obtained from wall tracking measurements are affected by the physics of the ultrasonic image formation and should be interpreted with care when linking them to the mechanical properties of the wall tissue [5].

As wall tracking is typically applied to more complexly deforming blood vessels like the carotid artery, we anticipate that its intricate wall mechanics will even further complicate the analysis. Therefore, simulated distension and strain estimates (circumferential and radial components) derived from an ultrasonic wall tracking algorithm will be compared with the true mechanical deformation and complex 3D strains as known from the FSI-simulation. Finally, simulation results will also be tested against wall tracking data acquired in 10 healthy subjects which will be further processed to yield circumferential and radial strain throughout the arterial wall.

\section{Methods}

\section{A. Multiphysics simulations}

We previously developed a simulation environment to simulate synthetic ultrasound images based on fully known but arbitrary hemodynamics and vessel wall mechanics [5]. An advanced numerical modeling strategy (fluid-structure interaction (FSI) simulations) was used to simulate the fluid motion 


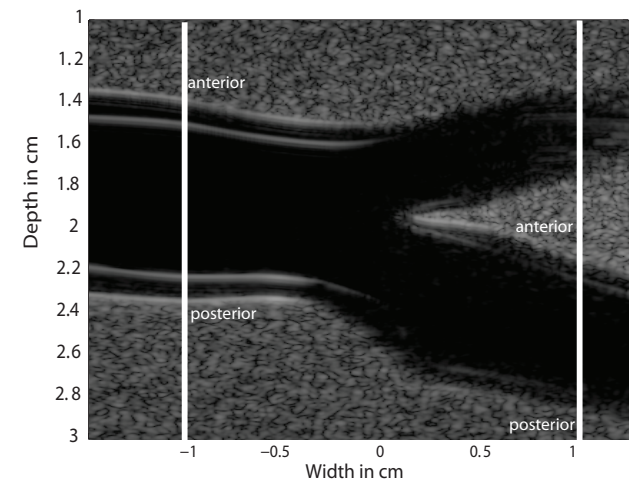

Fig. 1. B-mode image of the carotid artery showing the imaging setup for the wall tracking.

and vessel wall deformation of the vessel geometry under investigation. The applied FSI-code [5] was developed inhouse and couples 2 commercial solvers, one for the fluid (Fluent) and one for the structure (Abaqus). Next, the received RF-signals were obtained by integrating the FSI-results in the Field II ultrasound simulator, i.e. point scatterers representing blood and tissue were propagated according to the simulated arterial behavior. In this work, a patient-specific carotid artery model was investigated, as obtained after reconstruction from CT-scans of an 83-year old volunteer. A B-mode image of the carotid artery as resulting from these multiphysics simulations is shown in fig. 1. For more details on this simulation strategy, we refer to [5].

\section{B. Wall tracking}

Vessel wall velocities were estimated with a modified autocorrelation approach [4], allowing to determine vessel wall motion as: $\mathrm{z}[\mathrm{t}+\Delta \mathrm{t}]=\mathrm{z}[\mathrm{t}]+\mathrm{v} \Delta \mathrm{t}$, with $\mathrm{z}[\mathrm{t}]$ the position in the vessel wall, $v[\mathrm{t}]$ the estimated velocity, and $\Delta \mathrm{t}$ the velocity resolution corresponding to the packet size times the pulse repetition period $(3 \cdot 1 / 1000)$. An imaging setup with a high resolution was required and the $12 \mathrm{~L}$ linear array probe (GE Medical Systems, Milwaukee, WI, USA), as used in the applied distension software [4], was modeled with a 1.5 period sinusoidal pulse excitation of $8 \mathrm{MHz}$ centre frequency. Estimator performance was investigated in the presence of white noise, with a signal-to-noise ratio (SNR) of $-15 \mathrm{~dB}$ applied during post-processing of the RF-signals. The RFsignal from the surrounding tissue and fluid domain was neglected for this application.
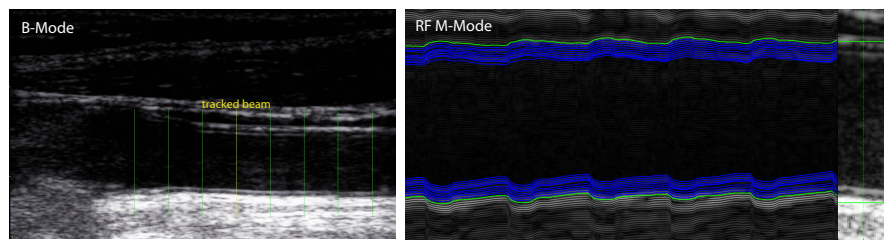

Fig. 2. Tracking method for in-vivo data.
By tracking the wall motion on both the anterior and posterior side of the blood vessel, the diameter distension curve can be determined as well as its associated distension measure $\Delta D=D_{\max }-D_{\min }$, with $D_{\max }$ and $D_{\min }$ respectively the maximal and minimal diameter during the cardiac cycle, obtained by tracking at a given depth in the vessel wall. Assuming planar deformation, radial $\left(\epsilon_{r r}\right)$ and circumferential strain $\left(\epsilon_{\theta \theta}\right)$ can be derived from the ultrasonic distension estimation $\Delta D$ as:

$$
\begin{aligned}
\epsilon_{r r} & =\frac{\partial \Delta D}{\partial D} \\
\epsilon_{\theta \theta} & =\frac{\Delta D}{D_{\min }}
\end{aligned}
$$

With $\partial D$ twice the distance between tracking points at a given depth in the vessel wall. Assuming a thin-walled tube and linear elasticity theory $\left(\sigma_{\theta \theta}=E \epsilon_{\theta \theta}\right.$ and $\left.\sigma_{\theta \theta}=\frac{\Delta P D_{\min }}{2 h}\right)$ and, small and planar deformations $\left(\epsilon_{\theta \theta}=\frac{\Delta D}{D_{\min }}\right)$, also the Young's modulus can be approximated from distension measurements as:

$$
E=\frac{\Delta p D_{\min }^{2}}{2 h \Delta D}
$$

with h the wall thickness and $\Delta p$ the systolic-diastolic pressure difference.

The wall tracking algorithm is based on an existing application where ultrasound beams are emitted at 8 different scan positions [4]. We performed distension analysis at 2 locations (in the common and internal carotid artery, cfr. fig. 1), and at 5 depths in the vessel wall. For the ground truth, the reference FSI-strains were calculated by applying equations 1 and 2 to the Abaqus node displacements. We also studied the complex $3 \mathrm{D}$ deformation nature of the blood vessel by visualizing the strains which Abaqus computes from a second order strain tensor obtained from large deformation theory. The ground truth for the Young's modulus is known from the input to the FSI-model.

\section{In-vivo analysis}

To verify our simulations, we analyzed a sample dataset drawn from a large population study conducted in 20022004, screening for early markers of cardiovascular disease in 2500 seemingly healthy adults (age 35-55 years) [6]. Part of the cardiovascular examination consisted of an ultrasonic wall tracking acquisition along 8 scanlines in the common carotid artery. We selected 10 consecutive samples from our database, the only criteria being that (i) the common carotid artery was sufficiently horizontal in the B-mode image, and (ii) the intima-media was clearly visible on both the anterior and posterior wall. These conditions were fulfilled in about $50 \%$ of the reviewed datasets. The dataset consisted of 5 men and 5 women, mean age $45.3+/-6.4$ years, height $167.3+/-$ $9.5 \mathrm{~cm}$, weight $71.5+/-10.2 \mathrm{~kg}$ and normal blood pressures (mean diastolic blood pressure $80.4+/-10.5 \mathrm{mmHg}$; mean systolic blood pressure $122.9+/-15.3 \mathrm{mmHg}$ ). We performed the tracking along the scanline well into the common carotid (far enough from the bulbus) showing the clearest intimamedia and media-adventitia transition in the B-mode image 

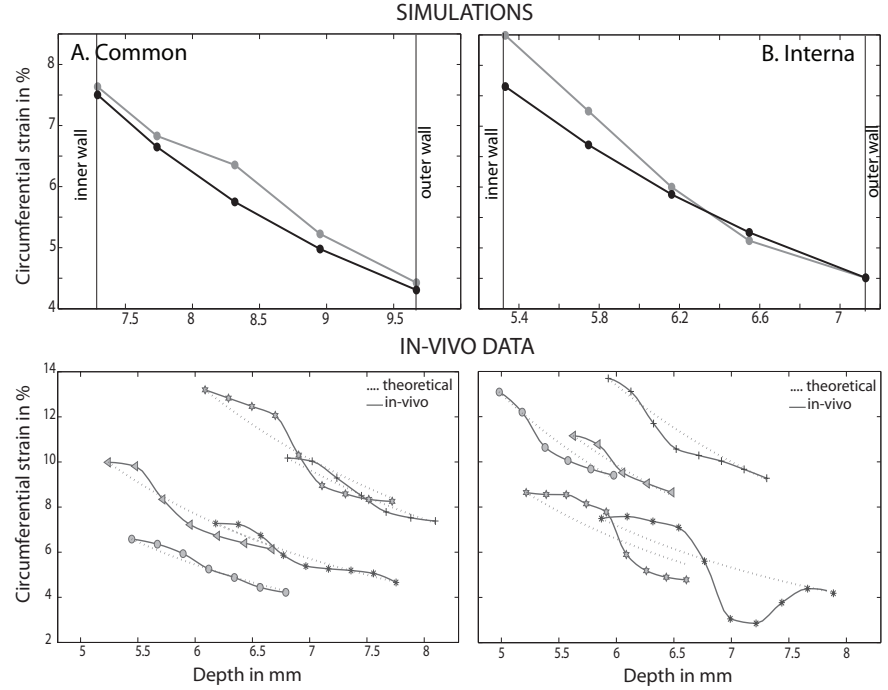

Fig. 3. Upper panels: simulated $\epsilon_{\theta \theta}$ in the common and internal carotid artery. Lower panels: $\epsilon_{\theta \theta}$ retrieved from 10 healthy subjects of the Asklepios population study.

(cfr. fig. 2). We adapted the wall tracking software, in order to start tracking from a designated point on the media-adventitia transition and from there on to track the wall movement every 100 micrometer towards the intima-media layer and lumen (cfr. fig.2). This tracking procedure is demonstrated in fig.2. We then applied formulas (1) and (2) to derive circumferential and radial strain from the in vivo data.

\section{RESULTS}

\section{A. Multiphysics simulations}

1) Common carotid: The circumferential strain $\epsilon_{\theta \theta}$ displayed as a function of the depth in the vessel wall (cfr. fig. 3 ), shows a decreasing trend in distension from inner to outer wall, both for the simulated ultrasound data (from $7.64 \%$ to $4.43 \%$ ) and the ground truth (from $7.50 \%$ to $4.31 \%$ ). While the reference curve shows a $1 / D^{2}$-trend, the ultrasound data has a slight sygmoid shape throughout depth, with a slight overestimation of $\epsilon_{\theta \theta}$ at the inner and outer wall (maximal absolute deviation throughout depth of $0.61 \%$ ). Much stronger deviations from the ground truth are found for the radial strain at peak systole throughout the vessel wall (data not shown), with a maximal absolute deviation of $5.44 \%$. The Young modulus as estimated from the distension measurements on the inner wall was $213 \mathrm{kPa}$, implying an underestimation of 14.62 $\%$ (note that the Young modulus derived from the Abaqus node displacements on the inner wall entailed an underestimation of $11.6 \%$ ).

2) Internal carotid: The relation between circumferential strain and depth is no longer a sygmoid, but still a decreasing trend from inner to outer wall is noticed, both for the simulated ultrasound data (from $8.49 \%$ to $4.51 \%$ ) and the ground truth (from $7.65 \%$ to $4.51 \%$ ) (cfr. fig. 3). The estimated Young modulus was $185 \mathrm{kPa}$, or an underestimation of $26.13 \%$.

\section{B. In-vivo data}

The lower panels of fig. 2 show the circumferential and radial strain for a female subject (age 39) with very good image quality (intima-media and media-adventitia transitions as clear as in the simulations, both at anterior and posterior side, cfr. B-mode image in upper left panel). The circumferential strain has a sygmoid shape and is plotted together with the theoretical relationship $\left(1 / D^{2}\right)$, derived using the conservation of mass principles [7]. The radial strain varies strongly and demonstrates a minimum (unrealistic) value of $-44 \%$. Data were fairly consistent in the dataset, as illustrated by the similar sygmoid trends in circumferential strain that were found in the other subjects (cfr. fig. 3). Radial strain curves are not shown for the other subjects due to their strongly oscillating nature and high inter-subject variability.

\section{Discussion}

There is a growing clinical interest in measuring arterial stiffness, and the uncomplicated nature of ultrasonic wall tracking measurements has gained a lot of attention in this domain. Many parameters based on arterial distension assessment have been investigated including distensibility, compliance, circumferential strain and different definitions of elasticity moduli (e.g. Young's modulus E, incremental elasticity modulus $E_{\text {inc }}$, stiffness index $\beta$ ). While these mechanical parameters have been widely assessed in clinical trials, it should be noted that they are based on the assumption of planar deformation of the artery. This might be valid in rather healthy and straight arterial segments, but not for more complexly deforming blood vessels like the carotid bifurcation. As such, it might be expected that wall tracking measurements and derived material parameters are inaccurate, a hypothesis we tested both with simulations and in-vivo data.

For the simulations, the circumferential strain assessed from ultrasound in the common carotid artery decreased from inner to outer wall, following a sygmoid-shaped trend throughout

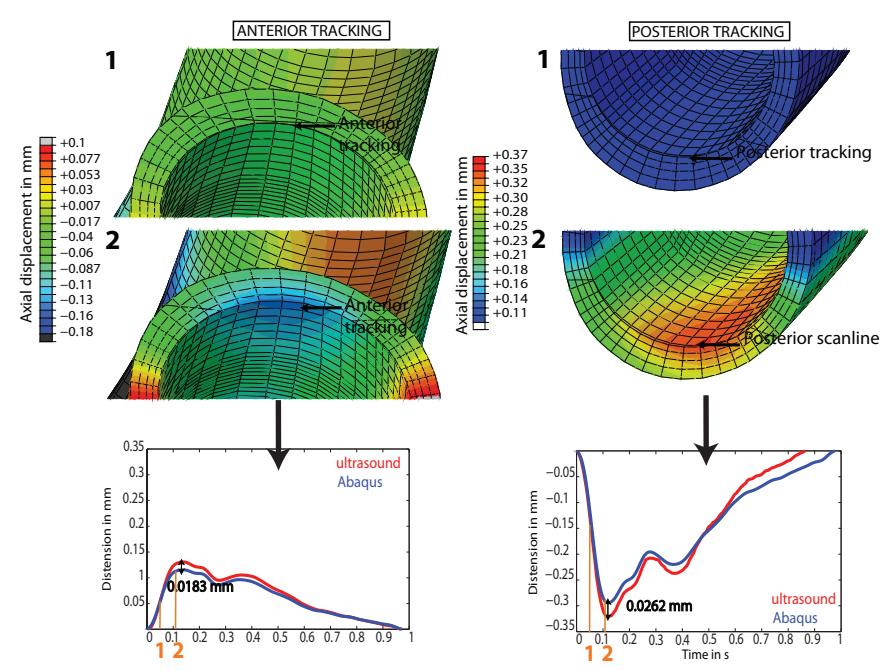

Fig. 4. Analysis of anterior and posterior tracking in the internal carotid artery. 


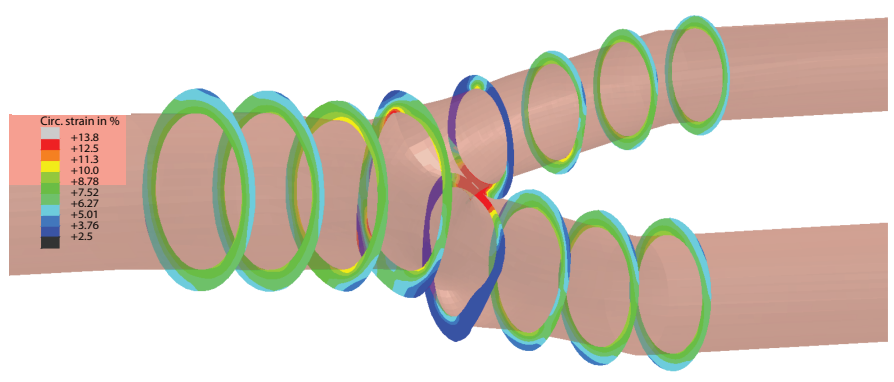

Fig. 5. Circumferential strain as obtained from large deformation theory in Abaqus, shown in 8 cross-sections at peak systole.

the vessel wall depth (in contrast with the expected $1 / D^{2}$ trend), which disappeared for the internal carotid artery (fig. 3). A similar sygmoid trend in circumferential strain was also retrieved in the common carotid artery of 10 seemingly healthy adults (fig. 3).

As we previously demonstrated for the simple and idealized case of a 3D tube [5], the sygmoid-shape finds it origin in the presence of specular reflections which cloud the measurement for sample points close to these reflections. Due to these strong reflections in the intima, the estimates in the inner wall are correlated and therefore the circumferential strain estimate is first overestimated. In the outer wall, the opposite occurs and the circumferential strain is underestimated. These effects are most visible in conditions where the vessel is perpendicular to the ultrasound beam, and when specular reflections are clearly present. Since the internal carotid artery is slightly tilted towards the ultrasound beam, the specular reflections disappear and as such, the sygmoid-shape in the circumferential strain curve disappears.

As wall tracking can be done for several material points within the arterial wall, data can be processed to obtain radial strain. Both our simulation and in-vivo data, however, indicate that results are not reliable. This estimate entails a spatial derivation of the distension data, and therefore amplifies inaccuracies in distension data. Inaccuracies in wall tracking will, most likely, also corrupt measurements of compressibility, which can be deduced from distension measurement at different depths in the arterial wall.

Interpretation of distension measurements is even further hampered when considering that the distension and strain estimates are derived from a tracking algorithm applied both to the anterior and posterior vessel wall, while obtaining just one strain estimate value for the complete cross-section at the end (cfr. fig. 4). For the simulated case, for instance, the anterior wall deformed less along the ultrasound beam, but more in the lateral and elevation direction. This might be misleading as both the anterior and posterior side might still have the same mechanical properties (the same E-modulus), as is the case in our FSI-simulation setup.

Although the FSI-Field II integration allows comparing simulated ultrasound data with the ground truth behind the image, one should carefully consider which reference data to use. In case of strain estimations, the ground truth in cfr. fig. 3 was the radial and circumferential strain as calculated from the Abaqus node displacements by equations 1 and 2. However, besides this self-calculated strain measure, we also visualized (at peak systole) circumferential logarithmic strain as obtained from large deformation theory in eight cross-sections of the carotid model (cfr. fig. 5), using a local cylindrical coordinate system for the common, internal and external carotid artery. Fig. 5 demonstrates that strain distributions are relatively uniform proximal and distal to the actual bifurcation, but strong local strain variations appear in the vicinity of the bifurcation itself, complicating a correct measurement from the relatively simple ultrasound wall tracking data. Note that these logarithmic strain values cannot be directly quantitatively compared to ultrasound estimations as the latter assumes small deformation theory.

\section{CONCLUSION}

To conclude, we demonstrated using both multiphysics simulations and in-vivo data that carotid strains (and in particular the radial component) estimated from wall tracking algorithms should be interpreted with caution. Both the complex 3D arterial deformation and the presence of specular reflections hamper the measurement, resulting in strain estimates deviating from the ground truth, with the error depending on the phase of the cardiac cycle and scanning location.

\section{REFERENCES}

[1] E. Hermeling, R. Reneman, and A. Hoeks, "Confluence of incident and reflected waves interferes with systolic foot detection of the carotid artery distension waveform.," Journal of Hypertension, vol. 26, pp. 2374-2380, 2008.

[2] H.Hansen, R.Lopata, and C. D. Korte, "Noninvasive carotid strain imaging using angular compounding at large beam steered angles validation in vessel phantoms," IEEE TMI, vol. 28, pp. 872-880, 2009.

[3] M. Couade, M. Pernot, C. Prada, E. Messas, J. Emmerich, P. Bruneval, A. Criton, M. Fink, and M. Tanter, "Quantitative assessment of arterial wall biomechanical properties using shear wave imaging," $U M B$, vol. 36 , pp. 1662-1676, 2010.

[4] S. I. Rabben, S. Bjaerum, V. Sorhus, and H. Torp, "Ultrasound based vessel wall tracking: an autocorrelation technique with rf center frequency estimation," Ultrasound in Med. \& Biol., vol. 28, pp. 507-517, 2002.

[5] A. Swillens, J. Degroote, J. Vierendeels, L. Lovstakken, and P. Segers, "A simulation environment for validating ultrasonic blood flow and vessel wall imaging based on fluid-structure interaction simulations: ultrasonic assessment of arterial distension and wall shear rate.," Medical Physics, vol. 37, no. 8, pp. 4318-4330, 2010.

[6] E. Rietzschel, M. De Buyzere, S. Bekaert, P. Segers, D. De Bacquer, L. Cooman, P. Van Damme, P. Cassiman, M. Langlois, P. van Oostveldt, P. Verdonck, G. De Backer, T. Gillebert, and A. Investigators., "Rationale, design, methods and baseline characteristics of the asklepios study.," $J$ Hypertension, vol. 22, pp. 973-981, 2004.

[7] P. Segers, S. I. Rabben, J. D. Backer, J. D. Sutter, T. Gillebert, L. V. Bortel, and P. Verdonck, "Functional analysis of the common carotid artery: relative distension differences over the vessel wall measured in vivo," J Hypertension, vol. 22, pp. 973-981, 2004. 